\title{
EL RETO DE UN CAMBIO INSOSLAYABLE. LA FORMACIÓN DEL PROFESORADO DE CIENCIAS
}

\author{
SÁEZ, M. J. \\ Escuela de Formación del Profesorado de EGB. Universidad de Valladolid.
}

\section{SUMMARY}

This work is an analysis of science teacher training needs according to the model put forward by the educational authorities and the problems arising in the school experimenting the new curricular design.

La Reforma Educativa que se perfila e impulsa desde instancias ministeriales y que, como parte de un cambio político más amplio, comenzó en la década de los 80 , es sin duda un reto para todos aquéllos que dedican sus esfuerzos a la enseñanza en los diferentes niveles del sistema educativo y también para toda la población, en la medida en que quizás la enseñanza junto con la sanidad son los dos aspectos dentro del sistema político que afectan más directa y extensamente a los ciudadanos.

La necesidad de renovación del sistema educativo no debe ser visto exclusivamente como el interés político de unos pocos, sino como la inexcusable necesidad de formar a ciudadanos que vivirán inmersos en una sociedad con una presencia cada vez mayor de la tecnología y por ende de los avances científicos que desde hace unas décadas se producen con una rapidez creciente. La masiva utilización de estos avances científicos y tecnologicos se convierte en un factor relevante en el mundo de la educación, dado que su uso está influyendo en las creencias y valores éticos y morales de los ciudadanos de hoy y no sólo por la necesidad de aprender a manejar estos elementos como herramientas indispensables.
En este marco, la contribución con nuevos enfoques en la formación del profesorado debe verse como un compromiso ineludible con el cambio que tiene que producirse, para que éste suponga una mejora de la formación de los ciudadanos en última instancia.

Por ello, me propongo analizar en este trabajo las necesidades de formación del profesorado específicamente en el área de Ciencias, y aunque tanto para la formacion inicial como para el reciclaje del profesorado, desde el punto de vista del objetivo a conseguir en el aula no es diferente, sí deben ser diferentes desde el modelo que deben adoptar, por el distinto punto de partida del que proceden unos y otros profesores. La experiencia docente, en el caso de los segundos, es vista por algunos autores como el elemento básico sobre el que debe pivotar la formación permanente, por ser importante ésta para conseguir cambios que afecten a las aulas.

Trataré, por tanto, de analizar la estrategia de la enseñanza/aprendizaje y el perfil del profesor de ciencias que se diseña en los documentos ministeriales, los problemas que se plantean en las clases de los centros 
piloto de la reforma y las características que deben tener los cursos de formación en función de ambas cosas.

\section{LOS DESEOS ESCRITOS}

Las necesidades de formación se encuentran en función del profesional que se desea, empecemos pues por delimitar el perfil de profesor que crean los documentos ministeriales y las peculiaridades propias del profesor de las llamadas ciencias experimentales. El "Vida escolar" publicado en 1984 como monográfico dedica * do a la reforma curricular de las enseñanzas obligato. rias, me parece un documento especialmente relevante, porque ha servido como impulsor/guía del proceso iniciado, con la fase experimental hoy ya prácticamen. te concluida, consciente de que el análisis de este documento no es más que el estudio de los deseos escritos, que sólo preparan para comprender cómo se implementa la reforma en las aulas.

El lenguaje que se utiliza tanto en éste como en otros documentos, sin duda refleja la colaboración a niveł ministerial de profesores de EGB y BUP en su elaboración y esta colaboración, importante para el análisis de las políticas educativas y para la aceptacion/difusión del "mensaje" fundamental que impuisa la reforma, presenta, como contrapartida, falta de rigor en el planteamiento que se pretende justificar. Refiriéndonos al campo concreto que aquí queremos tratar, a saber, la formación del profesorado de las llamadas ciencias experimentales o mejor, físico-naturales, esto puede verse en la siguiente afirmación: “...no se aprende ciencia escuchando, ni viendo como el profesor realiza un experimento para demostrar algo que ya ha explicado". La minusvaloración que se hace de la comunica ción oral en favor de lo que más tarde se califica como "hacer ciencia", significa no entender ésta como el elemento determinante del proceso de enseñanza/aprendizaje que se produce en las aulas. Y, por otro lado, aunque de menor importancia que lo anterior, la descalificación de la realización de demostraciones por parte del profesor que a veces resulta de gran utitidad no sólo como elemento motivador y recomendable en el caso de experimentos que entrañan algún riesgo y, por ello, desaconsejables para los estudiantes en estas edades que estamos tratando (12-16 años).

Sin embargo, situando el ejemplo dado en nuestro contexto escolar, también se puede interpretar que la idea que el documento quiere transmitir es la innegable necesidad de cambiar la estrategia de enseñanza, superando así la tradicional clase magistral a la que está acostumbrado nuestro profesorado. Pero lo que es admisible como crítica, no lo es como explicación de lo que pretende recomendar, que no es otra cosa, a mi entender, que la realización de clases en las que los alumnos participen activamente en el proceso de aprendizaje que tiene lugar en el aula y realicen actividades experimentales. En la base de este problema se encuentra, por tanto, el papel que juegan las actividades prácticas (el hacer ciencia) en la consecución del conocimiento básico.

Remitiéndonos a la naturaleza de la ciencia se pueden ver dos aspectos bien delimitados: 1) el conocimiento de conceptos y su interrelación, y 2) el método que el científico usa. En la enseñanza de las Ciencias deberíamos introducir a los alumnos en los dos tópicos, en el conocimiento que la ciencia nos proporciona, que deben entender y disfrutar, y también familiarizarles con el método de resolución de problemas con el cual el científico trabaja, y esto también con el fin de que les sea útil para su propia vida, y no sólo para que conozcan cómo se construye el conocimiento científico.

El trabajo práctico es una actividad que tiene lugar en el laboratorio y que como dice J.Solomon: "la ciencia pertenece al laboratorio como cocinar a la cocina y la floricultura al jardín". Sin embargo y a pesar de lo anterior, que es obvio, creo que hay necesidad de repensar cómo llevarlo a cabo, impulsar su uso indis. criminado sin una fundamentación clara y precisa, sólo puede conducir al ineficaz manejo de tiempo y recursos. Veamos un ejemplo: en una clase, los estudiantes siguen después de la explicación del profesor instrucciones de una ficha que explica cómo usar un mechero bunsen para calentar 3 recipientes iguales, con distintas cantidades de agua, durante el mismo tiempo y recoger los datos de las temperaturas antes y después de calentar. Siguiendo cuidadosamente esta operación, el profesor esperaba que los estudiantes entendieran la diferencia entre calor y temperatura. Al final de ia lección, preguntando a los estudiantes, se sacaba la conclusión de que la mayoría de éstos no habían apre* ciado tan sutil diferencia y muchos ni siquiera se habían dado cuenta de por qué calentaban el agua. Resultaba evidente que los alumnos no estaban actuando como los científicos resuelven los problemas, ni por supuesto aprendiendo física.

Analizando más detenidamente el significado que subyace a este planteamiento del trabajo práctico, vemos que implica que el conocimiento que se adquiere del proceso de la ciencia conduce inexorablemente al correcto entendimiento. Sin olvidar que la mayoría de las actividades experimentales son diseñadas con la intención de llegar a la respuesta correcta, el fín justifica los hechos y así, de facto, lo que se está proponiendo es que el conocimiento científico es objetivo y autónomo y así, el aprendizaje de las Ciencias se convierte en el hecho de que los estudiantes retengan lo que han descubierto otros y ellos desconocen. Preguntados los estudiantes, muchos opinan que este enfoque del trabajo práctico noes más que una sucesión de ejercicios con aparatos, que los debe conducir a resolver una pregunta que ni $\tan$ siquiera se han formulado.

En este caso, el trabajo práctico se ha diseñado con la idea fundamental de esclarecer la teoría. Un gran número de trabajos publicados (Holstein y Lunetta 1982, Osborne y Freyser 1985, Woolwough y AlIsop 1985), consideran ineficaz este tipo de planteamiento porque enseñar teoría a través del trabajo práctico resulta ineficaz a la hora de transmitir conceptos científicos a los estudiantes. 
Los documentos dicen también "Ios estudiantes deben realizar continuas y sistemáticas actividades similares a las desarrolladas por los científicos", y continúa "el método científico posibilita que los estudiantes cons. truyan sus propias estructuras conceptuaies, basándose en sus ideas como punto de partida". En mi opinión, en ambas afirmaciones el método científico sigue viéndose como algo "mágico" en el proceso de enseñanzal aprendizaje de las Ciencias Físico.Naturales. Al igual que otros autores, he argumentado en otras ocasiones que el método científico noes la panacea que soluciona los problemas de la enseñanza de las ciencias, que desde luego nos remite a cómo en nuestra cultura lo "científico" es la garantía de calidad, acierto y verdad de cualquier proyecto o idea, como Dios lo fue en siglos pasados (P. Tullier, "La trastienda del sabio").

En la estrategia de enseñanza/aprendizaje subyace en muchas ocasiones una errónea comprensión de lo que son los conocimientos científicos y en qué consiste el método científíco experimental. Y es que al enseñar Ciencias, se trabaja muchas veces con la idea implícita de que la ciencia es objetiva e independiente. Independiente el proceso también del que aprende y que, por tanto, el profesor sólo necesita de una buena técnica para implantar el conocimiento en la mente de los alumnos, a los que trata de guiar, en un ejercicio típicamente conductivista. Por otra parte, el enfoque que se da a la enser̃anza de las ciencias se basa en muchas ocasiones en cómo trabajan los científicos, asumiendo que éstos trabajan articulando los conceptos básicos y principios de la ciencia y conscientemente aplican éstos a la resolución de problemas. Al trabajar con esta estrategia se pretende que los chicos compren. dan los conceptos, para que éstos sean aplicados a nuevos problemas. Sin duda, la enseñanza de las ciencias debe potenciar en los estudiantes la destreza de resolver problemas, pero dudo que esta habilidad se alcance a través del aprendizaje y comprensión de un gran número de conceptos. En mi experiencia personal, una inadecuada relación entre la realización de actividades prácticas y la comprensión de los conceptos teóricos, aun admitiendo que sus objetivos no son distintos, el trabajo práctico queda totalmente desvir. tuado y la comprensión teórica no se logra. Por ello, mi opinión es que inicialmente deben separarse y establecerse el modo de actuación y la base de cada uno de ellos de forma no conjunta.

En el R.U., desde la innovación curricular emprendida a mediados de los 60 , se viene impulsando y practicando una estrategia que dedica gran parte del esfuerzo y del tiempo en las aulas al trabajo de laboratorio, realizado por grupos de alumnos (25). Los resultados de evaluar estas experiencia pueden ser muy útiles para orientar el enfoque de cuál es el trabajo de laboratorio más recomendable en nuestro contexto.

\section{Resumiendo esta experiencia:}

1. Hay que tener en cuenta la idea que los estudiantes tienen del trabajo práctico y los conocimientos que han adquirido cotidianamente (la TV en mi estudio se reveló como una fuente importante de ellos), evitando la tendencia existente entre el profesorado de ignorar este conocimiento de los estudiantes. La idea generalizada de que los estudiantes llegan a clase con la cabeza vacía, en lo que a conocimientos científicos se refiere, es falso como comprensión de la situación, y educativamente desastroso. Por ello, una de las etapas más importantes del proceso de enseñanza/aprendizaje debe ser ayudar a los chicos a articular y expresar, a través de la discusión, sus conocimientos previos. El profesor después de esto, estará en situación de proponer las actividades más adecuadas y los alumnos estarán en condiciones de realizar un trabajo práctico al que encontrarán significado y podrán ser capaces de seleccionar y dar sentido a la información que proporciona un experimento.

2. La discusión, representación y aplicación de la información se necesita independientemente de los aparatos y cacharros de laboratorio para crear una estructura cognitiva más madura. En este punto, Ia realidad deI Iaboratorio no contribuye al desarrollo y aplicación de conceptos abstractos en nuevos problemas teóricos. Dentro del marco de la teoría del "aprendizaje generado", Osborne y Wictrock (1983), mantienen que cuando se da información a los alumnos y se contestan sus preguntas, se les ayuda, pero esto no les conduce necesariamente a una nueva percepcion. Es en la discu* sión y en ia aplicación a problemas teóricos y prácticos, donde el alumno se capacita para usar la información, aumentando la comprensión y significado de los conocimientos teóricos. Allsop y Woolnough (1985) sugie ren que los estudiantes desarrollan la comprensión a través de la discusion, escribiendo, aplicando nuevas indagaciones y proponiendo soluciones más precisas. Estas indagaciones y experiencias les proporcionan comprensión y también les ayudan a recordar.

3. El trabajo experimental realizado en pequeños grupos puede satisfacer a través del lenguaje sus objetivos, porque los alumnos tienen la oportunidad de discutir el trabajo que están haciendo, lo que les ayuda a comprender los fenómenos y conceptos implicados.

Creo que es en este punto donde teoría y práctica deben encontrarse, dentro de un proceso de sucesiva forma. ción de ideas obtenidas a partir de las pasadas, articuladas a través de Ia aplicación y discusión y profundizando en experiencias prácticas más complejas que les lleven a un conocimiento superior. La idea difundida đe que sólo se enseñan ciencias si se realiza trabajo de laboratorio está lejos de ser lo correcto. En este planteamiento, la interacción urbana es fundamental a la hora de desarrollar en el alumno una vía personal en la comprensión del mundo.

En el siguiente diagrama, Allsop y Woolnough (1985) esquematizan muy acertadamente, en mi opinión, esta interaccion.

La idea que se plantea como aiternativa de la estrategia de enseñanza/aprendizaje, de "hacer ciencia" más que "enseñar ciencia", podría conducir a realizar experimentos de laboratorio sin otro objetivo que el de la 


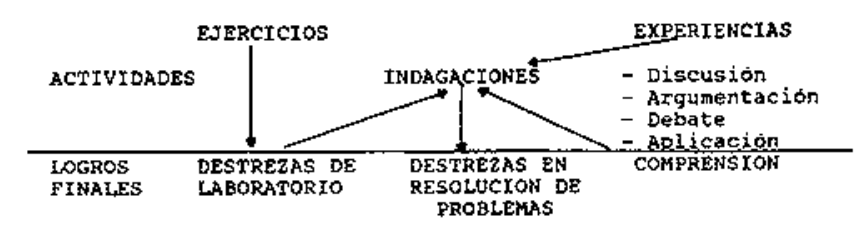

actividad y sin saber qué significa y su repercusión en términos de aprendizaje. Aceptar el "yo hago y entiendo" que supera el "yo hago y lo creo" tiene, en mi opinión, profundas implicaciones para enseñar cien. cias "haciendo ciencia", como he expuesto anteriormente al analizar la relación de trabajo práctico y contenidos térícos.

\section{EL PAPEL DEL PROFESOR DE CIENCIAS}

En afirmaciones tales como "...el profesor no debe enseñar ciencias, tiene que estimular a hacer trabajo científico" y en otras mencionadas más arriba, va implícito el rol que se pretende que desempeñe el profesor. Al igual que en otros países, en los procesos de cambio curricular se hace necesario definir nuevamente los papeles que profesores y alumnos deben jugar en el aula. En nuestro caso, lo que se está sugiriendo es que el profesor es el responsable de la creacion del ambiente adecuado para que el aprendizaje de los estudiantes pueda tener lugar. Cómo el profesor estimula y conduce a los estudiantes a aumentar sus conocimientos es lo que debe servir como base de las líneas metodológicas a seguir, así como la valoración de cómo el proceso de aprendizaje está teniendo lugar en cada uno de los alumnos.

Dado que el objetivo de este artículo es estudiar el contenido de los cursos de formación del profesor de ciencias, comentaré en este apartado algunos de los aspectos que deben incluirse en función del papel que el profesor de ciencias debe desempeñar.

Conviene tener en cuenta que los profesores dedicados a estas asignaturas tienen normalmente que asumir las tareas de preparación de las actividades de laboratorio, así como el mantenimiento de éste. A menudo, el problema en el laboratorio de ciencias no es sólo mantener la atención de los chicos en el trabajo experimental, sino también que el equipo y las actividades que están realizando funcionen adecuadamente.

Pero a parte de esta tarea específica del profesor de ciencias, éste debe trabajar las posibilidades que ofre* cen los aspectos educativos más básicos y centrar su trabajo:

- Tratando de presentar el tema y las actividades de tal forma que los alumnos puedan por ellos mismos reconstruir los problemas a resolver, y/o intentando presentar las tareas de aprendizaje como algo elegido por ellos y de su interés. Despertando la curiosidad por la ciencia.

- Estimulando a los alumnos a tomar la responsabili* dad de su propio aprendizaje.

- Estimulando a los alumnos a hacerse preguntas a ellos mismos y a otros y a centrar la atención sobre lo fundamental, iniciando un aprendizaje generativo que los conduzca a reflexionar sobre sus ideas y las de otros compañeros.

A menudo es difícil para el profesor, por la relación alumno-profesor que se mantiene en clase, profundizar en las ideas que tienen los chicos. Pocas recomendacio. nes se pueden hacer en este sentido, pero cuando una contestación inesperada se produce por parte de algún estudiante en una discusión, habitualmente se dedica poco tiempo a interpretar el sentido que ésta tiene. Descubrir y diagnosticar el conocimiento que los estu. diantes tienen sobre el tema que se está tratando consis. te, por un lado, en dejarles expresar sus ideas, pero esto solo no es suficiente, el profesor tiene que crear el clima en el cual pueda oir. El papel del profesor como "oyente" es inherente al del profesor que diagnostica los problemas del aprendizaje y de conocimientos que tienen los alumnos.

Existe también una tendencia marcada a cubrir etapas demasiado rápidamente, yendo a través de los hechos, sin dar tiempo a los estudiantes a que cojan el sentido de los conceptos en el mundo en el que viven. Los alumnos necesitan ser guiados en el proceso de unir las ideas existentes en la memoria (a largo plazo) y el intento de generar nuevas ideas o conceptos, para que sea almacenado en la memoria.

La génesis de conceptos nuevos para el estudiante pasa por desarrollar estrategias que les permitan procesar de forma efectiva la información que reciben y, de esta manera, conocer a dónde van y cómo llegar allí. Por eso es función también del profesor:

- Señalar los errores "lógicos" del pensamiento de los alumnos, los razonamientos inconsistentes y los argumentos que se basan en asunciones falsas.

- Hacerles darse cuenta de dónde están generalizando incorrectamente, o no lo están haciendo y es posible hacerlo.

- Ayudarles a considerar interpretaciones alternativas ante un hecho o fenómeno.

Es también un punto importante, para que la enseñanza de las ciencia tenga éxito, ( $R$. Driver, comunicación oral B.E.R.A. 1987, Manchester) que el profesor se dé cuenta de que si no ha habido evidencias suficientes del uso de un concepto nuevo, los alumnos volverán a sus ideas pasadas. Las actividades en clase tienen que asegurar que los estudiantes usan los nuevos conceptos "enseñados", debiendo utilizarlos para explicar nuevos hechos.

Por eso, la formación de un profesor no puede consistir en proporcionar técnicas de cómo enseñar, porque las 
"destrezas" que debe poner en práctica no son "directamente" enseñables. Es más útil e interesante considerar la enseñanza como una actividad que se desarrolla en el tiempo y en constante experimentación a través de la propia carrera de enseñante. Esto supone aprender a valorar sistemáticamente cómo se está actuando y cuánto han aprendido los estudiantes como resultado de la propia enseñanza. De hecho, es habitual valorar el aprendizaje que han realizado los alumnos después de un conjunto de lecciones, sin tener en cuenta que lo que los estudiantes han aprendido está en función de la estrategia que el profesor ha seguido.

Para que se produzca una real innovacion curricular, es absolutamente necesario el cambio del papel del profesor y creo que éste puede concretarse en saber diagnosticar las ideas existentes en las mentes de los alumnos, retarles a pensar en los fenómenos desde nuevos puntos de vista, ayudarles a adoptar soluciones alternativas y estimular en ellos el pensamiento reflexivo como características fundamentales.

\section{LO QUE SUCEDE EN LAS CLASES Y LO QUE DICEN ALUMNOS Y PROFESORES}

Analizaré pués los datos más significativos que he recogido en el trabajo de campo, realizado en los centros piloto de la fase experimental de la reforma y que han influido en los cursos que diseño en el siguiente apartado.

La evaluación se realizó a demanda del profesoradoen un ambiente de ayuda a los nuevos planes de trabajo en clase. Es fácil percibir que se trabaja con la crítica de la estrategia de enseñanza/aprendizaje anteriormente utilizada como guía y esto resulta una escasa apoyatura para determinar la nueva estrategia a experimentar. Así, el profesorado habla de una estrategia por "descubrimiento" en la medida que reconoce que ellos ya no deben ser los únicos que proporcionen la información a los alumnos, sino más bien que son éstos los que deben intentar conseguirla, al menos en parte, autónomamente y utilizando una variedad de fuentes. Pero el profesor tiene muchas dificultades para seguir de cerca el trabajo que cada estudiante ha realizado y si la información recogida contesta la pregunta previamente planteada y en qué međida ésta es entendida. Aunque en muchas ocasiones es la ratio profesor/alumno la que dificulta seriamente esta labor. Esto se produce a veces también porque haciendo trabajo experimental, es muy difícil seguir los ritmos previstos; habitualmente se cambia a otro tema, a expensas del tiempo final de aclaraciones y recapitulación. Sobre lo que incide de alguna manera la presión social que sigue existiendo sobre el tema contenidos en detrimento de que finalice el proceso de aprendizaje. Se observa la tendencia a entender que lo más importante es la actividad experimental como algo que modifica y mejora sustancialmente la comprensión de los conceptos en las ciencias físico-naturales, a pesar de que en muchas ocasiones los experimentos planteados no son, ni tan siquiera en sí mismos, relevantes para entender tal concepto.

Resulta clarificadora la opinión de los niños entrevistados al respecto de la nueva estrategia adoptada, que la consideran como "aprender jugando", "porque da menos pereza ir al cole asî" y "porque es más fácil sacar el curso", lo que en mi opinión habla de estímulo y motivación fundamentalmente. Preguntados sobre en qué consiste la nueva estrategia seguida por sus profesores, mencionan poco el trabajo experimental y, sin embargo, enfatizan cosas como: "ahora hay que conseguir las cosas pensando", "se aprende lo mismo que antes, pero de distinta manera y tarda más en olvidarse". Existe acuerdo entre ellos en que los debates en clase son lo más novedoso e importante: "Al principio del año me daba vergüenza participar porque, si decía algo mal, los otros se reían de mí y además, porque hablaba mal en alto. Ahora ya he aprendido a hacerlo y los otros no se ríen de mí. En los debates ves más opiniones y te atreves a preguntar a los otros, que te explican las cosas que no entiendes con tus mismas palabras. Las preguntas al profesor ayudan también a aclarar las dudas. Los debates me ayudan a entender las cosas".

Es cierto que es necesario que los profesores tengan experiencia en el trabajo de laboratorio y creo que debe dedicarse un tiempo a ello en los cursos de formación, porque es crucial que se sientan preparados y seguros en este aspecto. Pero, en mi opinión, hay que tener en cuenta que el punto central de la comprensión se encuentra en el debate y no en el experimento y, por tanto, la comunicación entre alumno y profesor.

Haciendo un diagrama de relaciones de cómo ven los alumnos la estrategia que se está siguiendo, se puede interpretar así:

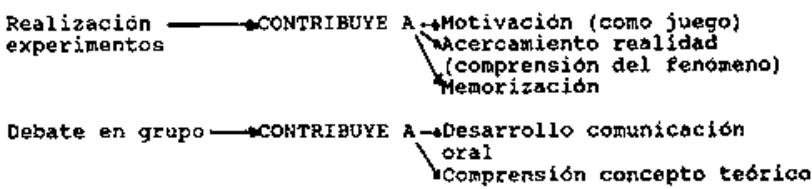

Quizás el otro aspecto relevante en esta estrategia es el trabajo en grupo, Io mencionaban los estudiantes rela. cionándolo con el aprendizaje y lo mencionan también los profesores. Por un Iado, los profesores se quejan de que "compañeros que tienen experiencias de años anteriores sobre aspectos que estamos trabajando no nos lo comunican, no hay oportunidad". Las reuniones de los profesores dentro de los colegios se dedican, en gran parte, para ponerse de acuerdo en horarios o en cómo compartir o distribuir el material de uso común. El trabajo en el departamento es algo más que un compartir los recursos disponibles, y es evidente que ésta es una de las tareas más difíciles de promover, dado que, para que se produzca, es necesario que exista algo común a tratar y que simultáneamente el producto de 
ese trabajo conjunto repercuta, de forma plausible, a encontrar soluciones a los problemas que se le presentan al profesorado. Lo común y útil es principalmente la discusión del proceso de enseñanza/aprendizaje que tiene lugar en las aulas.

Para terminar, otra opinión recogida es que el currículo de Ciencias sigue sin verse como una formación base, que contribuye a entender la base del sistema productivo en el que, lógicamente, la mayoría de nuestros escolares se verá envuelto en el futuro. La opinión de los alumnos a este respecto es que son los idiomas (el inglés) lo que les ayudará a encontrar trabajo; sin entrar en comentarios más profundos que se podrían hacer, señalar sólo que las ciencias que se les están enseñando están desconectadas del mundo de la empresa, de las fuentes de energía, de los sistemas de comunicación, etc.

\section{LAS CARACTERÍSTICAS DE LOS CURSOS DE FORMACION}

El informe de la OCDE, "Cursos de actualización y promoción de profesores, una condición para el cambio educativo", publicado en 1980 sobre cursos para profesores en activo, considera que hay que tener en cuenta que éstos no son la panacea del cambio, pero que para conseguir la máxima efectividad hay un aspecto que no debe perderse de vista y es que los cursos deben cubrir las necesidades de formación de los profesores individualmente y que, por ello, la liberación de tiempo para dedicarse a ellos es algo que debe tenerse en cuenta. En el Reino Unido, según el mencionado informe, los asesores de las autoridades locales trataron por todos los medios de conseguir que la mayoría del presupuesto se dedicara a la realización de distintos tipos de cursos de actualización más que en reducir el número (35) de alumnos por clase o en nuevas construcciones de edificios. En un estudio de este mismo tipo, realizado por Fullan (1987) en Canadá, a partir de estudios de caso, plantea como recomendación que los recursos deben utilizarse para atender a las necesidades de los profesores en el cambio curricular y centrarse en la reorganización de la escuela donde éstos desarrollan su labor.

Por todo lo dicho anteriormente, creo que sería lo más adecuado desarrollar cursos de formación en torno al currículo, entendiendo por éste: “...el intento de comunicar los principios esenciales y características de un proyecto educativo en una forma tal que esté abierto a la crítica y capaz de ser eficazmente trasladado a la práctica" como L. Stenhouse expuso en 1975, o como R. Walker plantea comentando la definición anterior: "Esta noción de currículo es algo más que la especifi. cación de los contenidos y requiere necesariamente tratar aspectos organizativos, así como otros problemas; es el que presenta un papel determinado a jugar para los profesores en el que los profesores no son sólo simples "instructores", sino que tienen que desarrollar necesariamente un enfoque más activo de su trabajo" (1987).
Está ampliamente asumido por toda la comunidad científica dedicada a la educación que el cambio curricular depende en gran medida de lo que los profesores sean capaces de modificar en sus clases. L. Stenhouse reco. nocía que: "Incluso aunque la enseñanza sea una profesión voluntariamente escogida, y no siempre es así, es difícil a veces mantener el acto de voluntad por el que una persona se somete a un empeño mantenido y arduo. El profesor no se interesa sólo por la justificación del conocimiento, tiene que estar motivado e inmerso en situaciones sociales que conduzcan al trabajo. Las dotes de mando son necesarias y mantener la autoridad, inevitable. El problema estriba en diseñar un esquema practicable de enseñanza que mantenga la autoridad, el mando y la responsabilidad del profesor, pero que simultáneamente no transmita el mensaje de que tal autoridad es la garantía del conocimiento".

Los cursos de formación que atañen a profesores en activo y a estudiantes de formación inicial, deben tener características distintas como ya mencioné más arriba, pero ambos tienen que resolver el mismo problema, "¿Como aprenden los profesores?" y, en definitiva, en qué consiste la inteligencia práctica. En opinión de J. Elliott (1979), el aprender a realizar acciones no se deriva necesariamente de principios téricos acerca de lo que es la práctica, sino que éstos se derivan necesariamente de la práctica. Afirma que las teorías implícitas en la práctica concreta pueden ser hechos generalizables, más que aspectos incluidos en teorías formales dentro de disciplinas académicas y que probablemente no van asociadas a un contexto sustantivo. Para Elliott, son abstracciones provenientes del conocimiento práctico, que se encuentran en actos concretos. El asumir esta idea implica dos aspectos importantes, a saber:

- Que el conocimiento práctico no puede reducirse a la aplicación consciente de principios, porque esto no significa en última instancia cómo la gente aprende a adquirir y desarrollar destrezas prácticas.

- Que genuinos y teóricos aspectos acerca de práctica no pueden ser aprendidos a priori. No se entiende primero un principio teórico acerca de la educación o enseñanza y más tarde se aplica al análisis de la práctica, porque es la comprensión lo que surge del análisis. Este enfoque significa asumir el método inductivo más que el deductivo y desarrollar teoría desde el estudio de un contex to determinado, que es en el que los profesores aprenden; considera además que la prác. tica no se deriva del conocimiento teórico, aunque éste, evidentemente, contribuye al desarrollo del conocimiento práctico.

Siendo coherente con lo anteriormente expuesto, el enfoque debe consistir en analizar la acción de los profesores usando los conceptos y teorías de acuerdo con las necesidades de comprensión surgidas de la práctica, más que aquéllas que son importantes en una disciplina concreta de conocimiento como la sociología, psicología, etc. El asunto consiste pues, en encontrar el adecuado equilibrio entre lo que debe ser prác. tico y el estudio de los aspectos teóricos que posibíliten analizar lo más relevante que surge de la acción, en el contex to específico. 
Para Schwab (1977), un problema práctico es sentido en primera instancia como un obstáculo en la acción sin necesariamente ser capaz de poderle definir; esta situacion puede resultar motivadora para el profesor e impulsarle a buscar datos acerca del problema. EI examen, estudio y análisis de estos datos conduce a hipótesis que ayudan a definir el problema. La búsqueda de más datos para apoyar o rechazar estas hipótesis hacen que el proceso se convierta pronto en algo dinámico y tremendamente abierto con respecto a su final. El análisis de una actividad puede contribuir a su realizacion inteligentemente al indicar en qué dirección mejora esta actividad.

Por eso creo que debe utilizarse en estos cursos, tanto en los de formación inicial como en los de reciclaje, la investigación en acción como estrategia de aprendizaje. L. Stenhouse (1981) mantenía que no había desarrolio curricular sin desarrollo profesional, por lo que es necesario pensar en términos de carrera profesional y experiencia adquirida y asumir que el aprendizaje de la práctica es parte de la práctica misma. R. Walker (1987) dice que: "Promover el cambio del papel de los profesores precisa que éstos desarrollen conocimientos acerca de la relación entre la acción, el contexto y ellos mismos. Que para que el conocimiento adquirido se convierta en acción es necesario conocerse a sí mismo. El conocimiento de sí mismo afecta a la autoestima y a la confianza en sí mismo. El desarrollo de la capacidad en el conocimiento de sí mismo requiere que el proceso vaya de lo más sencillo a lo más difícil, siguiendo las correspondientes etapas:

- Conocer las propias acciones y cómo éstas influyen en los ambientes de aprendizaje.

- Evaluar en la medida que concierne a la responsabilidad del profesor lo que sucede en las situaciones de aprendizaje de los estudiantes".

La reestructuración del sistema en base al desarrollo profesional se realiza en términos de la calidad de la enseñanza y es "personal" y "concreta", porque está relacionada con el formato del propio aprendizaje.

Otros muchos autores apoyan esta idea y así M. Conelly (1987) asevera que "... los profesores necesitan aprender sobre ellos mismos para aprender sobre su profesión". Para que este conocimiento de uno mismo que nos habla de la propia acción (educativa) y posibi* líta el cambio, es necesario poner en marcha el tan didáctico proceso de la reflexín. Si el objeto de estu. dio es la propia aula, es obvio que existe la posibilidad ai menos de conocerse a sí mismo. Si somos investigadores de la experiencia práctica, podemos llegar a saber más acerca de lo que sucede en el complejo mundo del aula y en cómo las personas llevan su conocimiento práctico a las interacciones con los otros y acerca de la naturaleza dialéctica de la investigación teorica.

Para J. Clandinin (1983), la clave se encuentra en entender la reflexión como un proceso y comprender que todo aquello que hacemos y decimos en clase es algo creado por nosotros mismos. En este tipo de aprendizaje reflexivo se enseña a hacer lo que se quiere conseguir ejemplifícándolo y analizándolo en la propia actuación, se ayuda a los estudiantes a reconocer los propios errores e ignorancias y se trabaja con ellos con la intención de que lo puedan desarrollar autónomamente y en colaboración con otros en el futuro. Se estimula la forma de aprendizaje por comparación y se asume que el aprendizaje es un cambio no sólo inherente al conocimiento, sino también a la acción. Sin olvi* dar que los juicios formados como resultado de la propia reflexión en la experiencia de las aulas no proporciona reglas generales o estándars de aplicación general.

\section{LOS CURSOS PARA PROFESORES EN ACTIVO}

La primera consideración a hacer es que éstos son, ante todo, cursos con una finalidad de utilidad para cubrir las necesidades y expectativas de las personas individuales en un mundo cambiante, donde los valores también cambian en el tiempo.

Desde el punto de vista del conocimiento práctico, los cursos para profesores en activo son un caso particular de desarrollo profesional de los que los ponen en práctica. En la experiencia adquirida por los estudiantes (profesores) que hacen estos cursos, sabemos que se produce una profunda experiencia de cambio. La inter* nalización de los principios teóricos de cambiar algo nuevo es fundamental a la acción de llevarlo a cabo y surge de la interacción de la apícación a la práctica, la reflexión y el análisis de la teoría que lo sustenta.

M. Conelly (1987), afirma, basándose en 20 años de expeziencia, que el mejor sitio de estudiar el currículo es en uno mismo, lo que significa que la acción profesional se encuentra en primer lugar en el conocimiento práctico del profesor y no en el conteniđo de los cursos que se imparten. Señala también que según aumenta la confianza en uno mismo, los profesores se encuentran en mejores condiciones de abordar más amplias y generales cuestiones profesionales. El profesor con experiencia va desarrollando progresivamente la capa cidad de abordar la naturaleza de la ciencia a estudiar, su estructura y organización, la naturaleza del aprendizaje, etc. El curso descrito de esta manera es, en efecto, una investigación aplicada a la práctica educativa con la idea de mejorar la situación. Por eso es necesario que, a través de la estrategia del conocimiento personal, se trabajen dos tipos de actividades encaminadas a suplir los aspectos que se desean desarrollar con respecto a la enseñanza de las ciencias, y que son por un lado:

- Las dificultades que presentan: temas nuevos que deben introducirse en el programa, enfoques más aplicados de temas que ya se encontraban en el programa y la realización de experiencias prácticas para ambos. Para tratar este primer punto, se realizará un trabajo de revisión bibliográfica y un ejercicio de entrevista con un científico que, por su especialidad, pueda dar una 
perspectiva actual del tema que se estudia y/o con una industria o entidad que represente una aplicación interesante en el sector productivo. Se deberán cubrir también unas horas en los laboratorios donde tendrán Ia oportunidad de realizar experimentos que ellos sugieren o que se consideran fundamentales para el tema que están trabajando. La valoración de este ejercicio, que se concluirá con la presentación y discusión por parte de todo el grupo, se realizará de forma anónima para todos los participantes, dando lugar a un ejercicio de valoración del aprendizaje específico en ciencias físico-naturales, que se realizará a través de seminarios. La realización de una programación para el aula del tema estudiado deberá presentarse como un ejercicio de planificación y organización que deberá ponerse en práctica.

- Como segunda actividad y con un período intensivo de seminarios, se trabajarán temas relacionados con el modus operandi de la investigación educativa, con la base del profesor como investigador, tratándose puntos tales como:

1. Qué preguntas hacerse para diseñar una investigación en el aula.

2. El estudio de casos.

3. EI trabajo de campo: la recogida de datos.
4. EI almacenaje, organización y análisis de los datos.

5. El ejercicio de la reflexión.

6. Cómo se escribe un informe.

Los tópicos arriba mencionados serán tratados simultáneamente a un ejercicio práctico que dé pauta a las discusiones del trabajo en grupo, así como a la introducción de los aspectos teóricos que se estudiarán.

El seminario se orienta a facilitar el acto de escribir acerca de los hechos educativos de los profesores, porque entiendo que la escritura no debe verse sólo como una forma de comunicación, sino como una forma de pensar y reorganizar las propias ideas. Importante, por tanto, el ámbito del propio conocimiento y del ejercicio de la reflexión.

Otro asunto interesante en este planteamiento es cómo evaluar la eficacia que el curso tiene y el impacto que con él se consigue. Se puede decir que existe prácticamente acuerdo dentro de la comunidad científica al respecto del tipo de evaluación que debe practicarse y esto es en cuanto a la participación del profesorado en ella. Se recomienda que sea formativa y que se centre tanto en eI desarrollo del curso, como en el impacto que ésta tiene en la estrategia de enseñanza-aprendizaje de los participantes.

\section{REFERENCIAS BIBLIOGRÁFICAS}

CLANDININ, I., 1986. Classroom practice. Teachers image in action. (Palmer Press: London).

CONNELLY, F.M., 1987. Becoming a teacher: a narrative approach of the education of teachers. Proxima publicación en Curriculum studies.

CONNELLY, M.F. y CLANDININ, I., 1986. On narrative method, personal philosophy and narratives unities in the story of teaching, Journal Research in Science Teacher, Vol. 23(4).

DRIVER, R. y BELL, B., 1986. Students thirkingand the learning of science: a constructiviste view, Sec. Science Review, Vol. 43(19).
DRIVER, R. Y ERICKSON, G., 1985. Theories in action: Some theoretical and empirical issues in the study of the students conceptual framework in science. (Leeds Uni. Press Leeds).

DRIVER, R., 1986. A constructivist approach to curriculum development in sciencie. Studies in Science Education, 13, p. 195.

ELLIOT, J., 1982. How do teachers learn? Inservice education and training of teachers. (CERI: París).

ELLIOT, J. y ADELMAN, C., 1981. Action research in classroom. (ford Teaching Project Cambrigde). 
FULLAN, M., 1982. The meaning of educational change. (Teachers College Press: New York).

FULLAN, M. y CONNELLY, M., 1987. Teacher education in Ontario: current practice and options for the future. Project Funded Ministry of Education. Canada.

HOLSTEIN, A. Y LUNETTA, V., 1982. The role of the lab. in science teaching neglected aspects of research. Jour. of Edu. Res. Vol. 52(2) p. 201.

OLSON, J. Y WALKER, R., 1982. Innovation in the science curriculum. (Croom Helm: London).

OSBORNE, R. y WITTROCK, J., 1983. Learning science: a generative process. Sci. Edu.,67(4), p. 489.

OSBORNE, R. y GILBERT, 3., 1985. Some issues of theory in science education. (Uni. Walkato Press: N. Zeeland).

OSBORNE, R. y FREXBERG, P., 1985. Learning in science. (Heinemann Pub: London).

SÁEZ, M.J., 1989. About the curriculum innovation in Spain. Próx. publicación en Pers. Eval. in Edu. Chicago.

SÁEZ, M.J. y ELLIOT, J., 1988. La investigación acción en Espan̄a: un proceso que empieza. Próx. publicación Revista de Educación. Madrid.

SARASON, S., 1982. The culture of the school and the probtem of the change. (Allyn and Bacon: New York).
SCHÖN, D., 1983. The reflective practitioner. (New York Basic Books: New York).

STAKE, R. y col., 1987. Case studies in science education. (Project for the NSF. Washington).

STENHOUSE, L., 1987. La investigación como base de la enseñanza. (Morata: Madrid).

WALKER, R. y GOODSON, I., 1982. Telling tales. CARE Ocasional Pub: Norwich).

WALKER, R., 1987. Support for curriculum and professional development: a report to the Victorian State Board of Education. (Deakin Uni. Press: Victoria).

WARNING, M., 1979. Social pressure and curriculum innovation: a study of the Nuffield Foundation Science Teaching Project. (Mehuen: London).

WOOLNOUGH, B. y ALLSOP, T., 1985. Practical work in sciencie. (Cambrigde Uni. Press: London).

OECD Reports: 1978. Innovation in inservice education and training of teachers, practice and theory.

1980. Evaluating inservice training for teachers.

1982. Inservice education and training of teachers. A condiction for educational change. 